\title{
Risk prediction and clinical model building for lymph node metastasis in papillary thyroid microcarcinoma
}

This article was published in the following Dove Press journal:

OncoTargets and Therapy

24 August 2016

Number of times this article has been viewed

\author{
Dao-zhe Lin ${ }^{1, *}$ \\ Ning $\mathrm{Qu}^{2,3, *}$ \\ Rong-liang $\mathrm{Shi}^{2,3}$ \\ Zhong-wu Lu ${ }^{2,3}$ \\ Qing-hai ji ${ }^{2,3}$ \\ Wei-li Wu' \\ 'Department of Surgical Oncology, \\ Rui'an People's Hospital, Third \\ Affiliated Hospital of Wenzhou \\ Medical University, Wenzhou, \\ ${ }^{2}$ Department of Head and Neck \\ Surgery, Fudan University Shanghai \\ Cancer Center, ${ }^{3}$ Department of \\ Oncology, Shanghai Medical College, \\ Fudan University, Shanghai, People's \\ Republic of China \\ *These authors contributed equally \\ to this work
}

Abstract: The surgical management of papillary thyroid microcarcinoma (PTMC), especially regarding the necessity of central/lateral lymph node dissection, remains controversial. This study investigated the clinicopathologic factors predictive of lymph node metastasis (LNM) in patients diagnosed with PTMC. Multivariate logistic regression analysis was used for PTMC patients identified from the Surveillance, Epidemiology, and End Results database who were treated by surgery between 2002 and 2012, to determine the association of clinicopathologic factors with LNM. According to the results, a total of 31,017 patients met the inclusion criteria of the study. Final histology confirmed 2,135 (6.9\%) cases of N1a disease and 1,684 cases (5.4\%) of N1b disease. Our multivariate logistic regression analysis identified variables associated with both central LNM and lateral lymph node metastasis (LLNM), including a younger age ( $<45$ years), male sex, non-Hispanic white and other race, classical papillary histology, larger tumor size, multifocality, and extrathyroidal extension; distant metastasis was also significantly associated with LLNM. The significant predictors identified from multivariable logistic regression were integrated into a statistical model that showed that extrathyroidal extension had maximum weight in the predictive role for LNM. LLNM was validated to be a significant risk factor for cancer-specific death in Cox regression analyses, whereas central LNM failed to predict a worse cancer-specific survival according to our data. Therefore, we suggested that central lymph node dissection could be performed in certain patients with risk factors. Given the prevalence of LLNM in PTMC, a thorough inspection of the lateral compartment is recommended in PTMC patients with risk factors for precise staging; from the viewpoint of a radical treatment for tumors, prophylactic lateral lymph node dissection that aims to remove the occult lateral lymph nodes may be an option for PTMC with risk factors. Multicenter studies with long-term follow-up are recommended to better understand the risk factors and surgical management for cervical nodes in PTMC.

Keywords: thyroid cancer, microcarcinoma, regional metastasis, model, SEER, survival analysis

\section{Introduction}

In many regions of the world, the incidence of papillary thyroid cancer (PTC) is increasing more rapidly than that of any other malignancy. ${ }^{1}$ This increase may be explained by the increased detection of papillary thyroid microcarcinoma (PTMC), which is defined as a lesion of $\leq 10 \mathrm{~mm}$ in diameter. With the continuously increasing incidence of PTMC worldwide, the clinical care of PTMC has become a management dilemma, and more importantly, a public health issue. ${ }^{2}$ 
The management approach to PTMC cannot be overly cavalier because a significant percentage of patients with microcarcinoma have lymph node metastases (LNMs) at the time of presentation, which implies future recurrences, and a very small number of patients may have, or will subsequently develop, distant metastasis..$^{2-13}$ On the other hand, given the potentially high prevalence of LNM in PTMC, some authors agree that prophylactic lymph node dissection (LND) permits an improved staging of the disease that may guide subsequent treatment; ${ }^{14}$ however, few studies have demonstrated a meaningful role of prophylactic LND in relation to locoregional control or survival in PTMC. A systematic review that included 8,345 PTMC patients explored the risk factors associated with central lymph node metastasis (CLNM) and proposed that prophylactic central lymph node dissection (CLND) should be considered in PTMC patients with risk factors. ${ }^{15}$ However, the deficiency of long-term follow-up data for the analysis was a limitation in that study. Finally, for some groups, it appears reasonable to use a selective approach that applies CLND at the time of initial operation only to patients with clinically evident disease based on preoperative evaluation of primary tumor and nodal disease.

The present research aimed to investigate the frequency, pattern, and predictive factors for LNM in PTMC, and evaluate the optimal surgical treatments for regional nodes in PTMC over a long-term follow-up period, using data from the Surveillance, Epidemiology, and End Results (SEER) Program from 2002 to 2012. Finally, we developed a risk calculator based on risk factors to predict LNM risk, and we discuss the role of prophylactic LND in PTMC patients.

\section{Methods}

\section{Selection of patients from the SEER database}

For this study, data were extracted from the SEER cancer registry, which is a population-based registry sponsored by the National Cancer Institute which collects information on cancer incidence and survival from 17 population-based cancer registries, including $\sim 28 \%$ of the US population. ${ }^{16}$ The SEER data contain no identifiers and are publicly available for studies on cancer-based epidemiology and health policy. The National Cancer Institute's SEER*Stat software (Version 8.1.2; Surveillance Research Program, National Cancer Institute, Bethesda, MD, USA; www.seer. cancer.gov/seerstat) was used to identify patients who were diagnosed with single primary PTMC from 2004 to 2012. Patients who had undergone surgery for PTMC as the only malignancy or the first one of more malignancies were included. Histology types were limited to papillary cancer $(8,260)$ and papillary cancer follicular variant $(8,340)$. The patients with insufficient data or unknown clinicopathologic profile, undetermined histology, or other types of thyroid cancers (follicular thyroid cancer, medullary thyroid cancer, anaplastic thyroid cancer, etc) were excluded.

\section{Ethics statement}

This study was performed in compliance with the Declaration of Helsinki, approved by an independent ethics committee/ institutional review board at Fudan University Shanghai Cancer Center, and conducted in accordance with the approved guidelines. Data released from the SEER database do not require informed patient consent because they contain no identifiers and are publicly available. We obtained permission to access the research data file in the SEER Program from the National Cancer Institute (reference number 13579-Nov 2014).

\section{Assessment of clinicopathologic variables}

The following variables were used to analyze the risk factors for CLNM and lateral lymph node metastasis (LLNM): sex, age at diagnosis, race, maximal tumor size, multifocality, and extrathyroidal extension (ETE), surgical procedures, adjuvant therapies, and survival time were extracted from the SEER database. Race was categorized as African American, non-Hispanic white, and others (American Indian/Alaska [AK] Native, Asian/Pacific Islander) as provided by the SEER database ${ }^{17}$ According to the current staging system, an age of 45 years was used as the cutoff point to divide all patients into two groups: younger patients ( $<45$ years) and older patients ( $\geq 45$ years). ${ }^{18}$ Multifocal primary lesions were defined as two or more cancer sites within the thyroid; a solitary intrathyroidal primary lesion represented only a single cancer site within the thyroid. An ETE of the primary tumor consisted of thyroid capsule, minimal ETE (strap muscle), and gross ETE (nerves, esophagus, larynx, sternocleidomastoid muscle, etc). The multifocal lesion and ETE were both assessed in the final pathology. The end point of the present study was cancer-specific survival (CSS) of PTMC which was calculated from the day of diagnosis to the day of cancer-specific death and was shown as "SEER cause-specific survival" in the SEER database. We followed the guidance of the 2010 TNM classification of American Joint Committee on Cancer. ${ }^{17,18}$

\section{Statistical analysis}

The results are expressed as n (\%) or mean \pm standard deviation. The statistical analyses were performed using Student's 
$t$-test, the $\chi^{2}$ test, or the Mann-Whitney test as appropriate. The odds ratio (OR) and the $95 \%$ confidence interval (CI) for the relationships between each variable and LNM (yes or no) were calculated using binary logistic regression. A final model selection was performed by a backward stepdown selection process with the Akaike information criterion. ${ }^{19}$ The discrimination and calibration of the simplified scoring system were then assessed and internally validated using bootstrapping techniques. A Cox proportional hazards regression model was built to evaluate the effects of variables on cancer mortality in PTMC patients and then adjusted by multivariate analysis for patient characteristics and tumor characteristics. The hazard ratios for relationships between each variable and CSS were calculated using a binary Cox regression model. All statistical analyses were performed using package of $\mathrm{rms}^{20}$ in $\mathrm{R}$ version 3.2.2 (The R Foundation, Vienna, Austria; http://www.r-project.org/). Two-sided $P<0.05$ was considered statistically significant.

\section{Results}

\section{Baseline characteristics}

We identified 75,791 eligible patients with PTC from the SEER database treated between 2002 and 2012; of these patients, 31,017 (40.9\%) were diagnosed with PTMC histologically. The age of the enrolled patients ranged from
3 to 99 years (mean 50.9 \pm 13.9 ). Regarding surgical procedures, $23,572(76.0 \%)$ patients underwent total thyroidectomy (TT). Of the patients who underwent TT, radioisotope therapy was performed in 8,886 cases (28.6\%) as an adjuvant therapy. Among the patients who underwent LND, the final pathological results revealed that 2,135 patients $(6.9 \%)$ had positive malignant LNM in central neck, while 1,684 (5.4\%) had it in lateral neck. The clinicopathologic characteristics of all patients are summarized in Table 1 .

\section{Risk factors for CLNM}

The primary tumor size as a continuous variable was significantly different between the CLNM-negative and CLNM-positive groups ( $P=0.001$, Table 1$)$. Tumor size was associated with CLNM according to a univariate logistic regression (OR 1.199, 95\% CI 1.181-1.219, $P=0.001$ ). Other variables, such as male sex (OR 0.675, 95\% CI 0.609-0.749, $P=0.001)$, younger age $(<45$ years, OR $0.646,95 \%$ CI $0.591-$ 0.706, $P=0.001$ ), non-Hispanic white (OR 1.978, 95\% CI 1.550-2.522, $P=0.001$ ) and other (American Indian/ AK Native, Asian/Pacific Islander) race (OR 2.152, 95\% CI 1.632-2.839, $P=0.001)$, classical papillary histology (OR $0.635,95 \%$ CI $0.569-0.708, P=0.001$ ), multifocality (OR 2.063, 95\% CI 1.888-2.253, $P=0.001$ ), and ETE (capsule, OR $1.537,95 \%$ CI $1.232-1.919, P=0.001$; minimal ETE,

Table I Characteristics of patients with PTMC with LNM identified from the SEER database

\begin{tabular}{|c|c|c|c|c|c|c|c|}
\hline Variable & Total & CLNM-negative & CLNM-positive & $P$-value & LLNM-negative & LLNM-positive & $P$-value \\
\hline Total & $31,017(100.0)$ & $28,882(93.1)$ & 2,135 (6.9) & & $29,333(94.6)$ & I,684 (5.4) & \\
\hline \multicolumn{8}{|l|}{ Sex } \\
\hline Male & $5,725(18.5)$ & $5,201(18.0)$ & $524(24.5)$ & 0.001 & $5,117(17.4)$ & $608(36.1)$ & 0.001 \\
\hline Female & 25,292 (8I.5) & 23,681 (82.0) & I,6II (75.5) & & $24,216(82.6)$ & $1,076(63.9)$ & \\
\hline \multicolumn{8}{|l|}{ Age (years) } \\
\hline$<45$ & $10,272(33.1)$ & $9,362(32.4)$ & $910(42.6)$ & 0.001 & $9,485(32.3)$ & 787 (46.7) & 0.001 \\
\hline$\geq 45$ & $20,745(66.9)$ & $19,520(67.6)$ & $\mathrm{I}, 225(57.4)$ & & 19,848 (67.7) & $897(53.3)$ & \\
\hline \multicolumn{8}{|l|}{ Race } \\
\hline Black & I,907 (6.I) & I,837 (6.4) & $70(3.3)$ & 0.001 & I,872 (6.4) & $35(2.1)$ & 0.001 \\
\hline White & $25,958(83.7)$ & $24,139(83.6)$ & I,819 (85.2) & & 24,5 I 8 (83.6) & I,440 (85.5) & \\
\hline Other ${ }^{\mathrm{a}}$ & $2,784(9.0)$ & 2,573 (8.9) & $211(9.9)$ & & 2,597 (8.9) & I87 (II.I) & \\
\hline Size $(\mathrm{mm})$ & $5.4 \pm 3.0$ & $5.3 \pm 3.0$ & $6.9 \pm 2.7$ & 0.001 & $5.4 \pm 3.0$ & $6.7 \pm 2.7$ & 0.001 \\
\hline \multicolumn{8}{|l|}{ Histology } \\
\hline FV-PTMC & $22,482(72.5)$ & 20,77। (7I.9) & I,7II (80.I) & 0.001 & $21,113(72.0)$ & I,369 (8I.3) & 0.001 \\
\hline Papillary & $8,535(27.5)$ & $8, I I I(28.1)$ & $424(19.9)$ & & $8,220(28.0)$ & $315(18.7)$ & \\
\hline Multifocality & $|0,75|(34.7)$ & $9,664(35.5)$ & I,087 (50.9) & 0.001 & $9,763(33.3)$ & $988(58.7)$ & 0.001 \\
\hline \multicolumn{8}{|l|}{ ETE } \\
\hline Capsule & $\mathrm{I}, 000(3.2)$ & $910(3.2)$ & $90(4.2)$ & 0.001 & $904(3.1)$ & $96(5.7)$ & 0.001 \\
\hline Minimal & I,643 (5.3) & I,330 (4.6) & $313(14.7)$ & & I,297 (4.4) & $346(20.5)$ & \\
\hline Gross & $161(0.5)$ & $135(0.5)$ & $26(1.2)$ & & $112(0.4)$ & $49(2.9)$ & \\
\hline Metastasis $^{b}$ & $92(0.3)$ & $81(0.3)$ & II (0.5) & 0.001 & $46(0.2)$ & $46(2.7)$ & 0.001 \\
\hline TT & $23,572(76.0)$ & 21,661 (75.0) & I,9।I (89.5) & & 21,979 (74.9) & I,593 (94.6) & 0.001 \\
\hline Radioisotopes & $8,886(28.6)$ & $7,585(26.3)$ & I,30I (60.9) & 0.001 & $7,688(26.2)$ & I, I98 (7I.I) & 0.001 \\
\hline
\end{tabular}

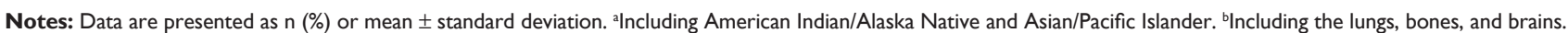
Abbreviations: PTMC, papillary thyroid microcarcinoma; SEER, Surveillance, Epidemiology, and End Results; LNM, lymph node metastasis; CLNM, central lymph node metastasis; LLNM, lateral lymph node metastasis; FV-PTMC, follicular variant of papillary thyroid microcarcinoma; ETE, extrathyroidal extension; TT, total thyroidectomy. 
OR 2.992, 95\% CI 1.961-4.566, $P=0.001$; gross ETE, OR $3.657,95 \%$ CI 3.203-4.175, $P=0.001$ ) were also found to be significantly associated with CLNM via the univariate logistic regression. Distant metastasis (OR 1.841, 95\% CI 0.979-3.462, $P=0.058$ ) was not associated with CLNM. When these variables were included in the multivariate logistic regression models, they were all found to be significant predictors of CLNM except distant metastasis (Table 2).

\section{Risk factors for LLNM}

The risk factors for LLNM were evaluated using univariate and multivariate logistic regression analyses for the patients who had positive LLNM according to the final pathological results. An increase in the primary tumor size was associated with a corresponding increase in the risk of LLNM (OR $1.112,95 \%$ CI $1.091-1.133, P=0.001)$. With the exception of the primary tumor size, the univariate analyses also indicated that male sex (OR 0.374, 95\% CI 0.337-0.415, $P=0.001)$, younger age $(<45$ years, OR $0.545,95 \% \mathrm{CI}$ 0.493-0.601, $P=0.001$ ), non-Hispanic white (OR 3.141, 95\% CI 2.239-4.407, $P=0.001$ ) and other (American Indian/ AK Native, Asian/Pacific Islander) race (OR 3.851, 95\% CI 1.971-5.868, $P=0.001)$, classical papillary histology (OR $0.591,95 \%$ CI $0.521-0.670, P=0.001)$, multifocality (OR $2.845,95 \%$ CI 2.575-3.145, $P=0.001$ ), and ETE (capsule, OR 2.405, 95\% CI 1.934-2.992, $P=0.001$; minimal ETE, OR 6.042, 95\% CI 5.295-6.895, $P=0.001$; gross ETE, OR 9.909, 95\% CI 7.048-13.931, $P=0.001$ ) were also found to be significantly associated with LLNM via the univariate logistic regression. In particular, distant metastasis was a strong predictor of LLNM (OR 17.880, 95\% CI 11.846-26.987, $P=0.001)$. When these variables were included in the multivariate logistic regression models, they were all found to be associated with LLNM (Table 3).

\section{Prognostic model for cervical LNM}

The final method of displaying the relationships among tumor size, other predictors, and probability of LNM is to construct a model. A predictive model not only sheds light on the effect of each predictor on the probability of response by levels of other factors but also allows quick estimation of probability of response for individual subjects. ${ }^{21}$ For convenience, we define the response to be $Y=0$ or 1 , with $Y=1$ denoting positive development of LNM. The significant predictors identified from multivariable logistic regression were integrated into a statistical model that shows the relationship between tumor size and LNM occurrence in PTMC patients (Figure S1). ${ }^{21}$ The values of the predictors derived from the coefficients of logistic regression, and the regression equation for LNM risk are given by the following equations:

$$
\operatorname{Prob}\{\mathrm{CLNM}=1\}=\frac{1}{1+\exp (-X \beta)},
$$

where

$$
\begin{aligned}
X \hat{\beta}= & -2.58+0.14 \text { race }-0.39 \text { sex }-0.47 \text { histology } \\
& +0.15 \text { size }+0.18 \text { capsule }+0.86 \text { minimal ETE } \\
& +0.66 \text { gross } \text { ETE }+0.51 \text { multifocality }-0.38 \text { age }
\end{aligned}
$$

\begin{tabular}{|c|c|c|c|c|}
\hline \multirow[t]{2}{*}{ Independent variable } & \multicolumn{2}{|l|}{ Univariate } & \multicolumn{2}{|l|}{ Multivariate } \\
\hline & OR (95\% Cl) & $P$-value & OR (95\% Cl) & $P$-value \\
\hline Sex (female vs male) & $0.675(0.609-0.749)$ & 0.001 & $0.673(0.605-0.748)$ & 0.001 \\
\hline Age ( $\geq 45$ vs $<45$ years) & $0.646(0.59 I-0.706)$ & 0.001 & $0.683(0.623-0.749)$ & 0.001 \\
\hline \multicolumn{5}{|l|}{ Race } \\
\hline Black & I (reference) & & I (reference) & \\
\hline White & $1.978(1.550-2.522)$ & 0.001 & $1.656(1.295-2.117)$ & 0.001 \\
\hline Other $^{\mathrm{a}}$ & $2.152(1.632-2.839)$ & 0.001 & $1.578(1.191-2.089)$ & 0.001 \\
\hline Size & $1.199(1.181-1.219)$ & 0.001 & $1.172(1.153-1.191)$ & 0.001 \\
\hline Histology (PTMC vs FV-PTMC) & $0.635(0.569-0.708)$ & 0.001 & $0.64 \mid(0.574-0.7 \mid 6)$ & 0.001 \\
\hline Multifocality & $2.063(1.888-2.253)$ & 0.001 & $1.662(1.5|6-1.82|)$ & 0.001 \\
\hline \multicolumn{5}{|l|}{ ETE } \\
\hline Negative & I (reference) & & I (reference) & \\
\hline Capsule & 1.537 (1.232-1.919) & 0.001 & $1.232(1.183-1.543)$ & 0.007 \\
\hline Minimal & $2.992(1.96 I-4.566)$ & 0.001 & $2.119(1.377-3.263)$ & 0.001 \\
\hline Gross & $3.657(3.203-4.175)$ & 0.001 & $2.546(2.218-2.921)$ & 0.001 \\
\hline Metastasis $^{\mathrm{b}}$ & I.84| (0.979-3.462) & 0.058 & I.I52 (0.598-2.222) & 0.672 \\
\hline
\end{tabular}

Table 2 Univariate and multivariate logistic regression analysis for CLNM in patients with PTMC

Notes: ancluding American Indian/Alaska Native and Asian/Pacific Islander. Including the lungs, bones, and brains.

Abbreviations: CLNM, central lymph node metastasis; PTMC, papillary thyroid microcarcinoma; OR, odds ratio; Cl, confidence interval; FV-PTMC, follicular variant of papillary thyroid microcarcinoma; ETE, extrathyroidal extension. 
Table 3 Univariate and multivariate logistic regression analysis for LLNM in patients with PTMC

\begin{tabular}{|c|c|c|c|c|}
\hline \multirow[t]{2}{*}{ Independent variable } & \multicolumn{2}{|l|}{ Univariate } & \multicolumn{2}{|l|}{ Multivariate } \\
\hline & OR $(95 \% \mathrm{Cl})$ & $P$-value & OR $(95 \% \mathrm{Cl})$ & $P$-value \\
\hline Sex (female vs male) & $0.374(0.337-0.415)$ & 0.001 & $0.360(0.322-0.40 I)$ & 0.001 \\
\hline Age ( $\geq 45$ vs $<45$ years) & $0.545(0.493-0.601)$ & 0.001 & $0.503(0.453-0.559)$ & 0.001 \\
\hline \multicolumn{5}{|l|}{ Race } \\
\hline Black & I (reference) & & I (reference) & \\
\hline White & 3.141 (2.239-4.407) & 0.001 & $2.44 \mathrm{I}(1.730-3.443)$ & 0.001 \\
\hline Other $^{a}$ & $3.85 I(1.97 I-5.868)$ & 0.001 & $2.620(1.802-3.808)$ & 0.001 \\
\hline Size & $1.170(1.150-1.191)$ & 0.001 & $1.112(1.091-1.133)$ & 0.001 \\
\hline Histology (PTMC vs FV-PTMC) & $0.591(0.521-0.670)$ & 0.001 & $0.625(0.549-0.71 \mathrm{I})$ & 0.001 \\
\hline Multifocality & $2.845(2.575-3.145)$ & 0.001 & $2.265(2.039-2.517)$ & 0.001 \\
\hline \multicolumn{5}{|l|}{ ETE } \\
\hline Negative & I (reference) & & I (reference) & \\
\hline Capsule & $2.405(1.934-2.992)$ & 0.001 & $2.105(1.68 I-2.637)$ & 0.001 \\
\hline Minimal & $6.042(5.295-6.895)$ & 0.001 & $4.601(3.994-5.300)$ & 0.001 \\
\hline Gross & $9.909(7.048-|3.93|)$ & 0.001 & $7.093(4.910-10.246)$ & 0.001 \\
\hline Metastasis ${ }^{\mathrm{b}}$ & 17.880 (11.846-26.987) & 0.001 & II.948 (7.523-I8.975) & 0.001 \\
\hline
\end{tabular}

Notes: ancluding American Indian/Alaska Native and Asian/Pacific Islander. 'Including the lungs, bones, and brains.

Abbreviations: LLNM, lateral lymph node metastasis; PTMC, papillary thyroid microcarcinoma; OR, odds ratio; Cl, confidence interval; FV-PTMC, follicular variant of papillary thyroid microcarcinoma; ETE, extrathyroidal extension.

$$
\operatorname{Prob}\{\operatorname{LLNM}=1\}=\frac{1}{1+\exp (-X \beta)},
$$

where

$$
\begin{aligned}
X \hat{\beta}= & -1.58+0.21 \text { race, }-1.04 \text { sex },-0.51 \text { histology } \\
& +0.09 \text { size, } 0.13+\text { distant metastasis, } \\
& +0.70 \text { capsule, }+1.42 \text { minimal ETE, } \\
& +1.96 \text { gross ETE, }+0.81 \text { multifocality, } \\
& -0.67 \text { age distant metastasis, ETE, } \\
& \text { multifocality, }>45 \mathrm{yr}=1,0 \text { otherwise; } \\
& \text { race[black] }=1,[\text { white }]=2,[\text { other }]=3 \\
& \text { histology }[\mathrm{PTC}]=1,[\mathrm{FV}-\mathrm{PTC}]=2 .
\end{aligned}
$$

The output of validation indicates minor overfitting. Overfitting would have been worse had the risk factors not been so strong. The closeness of the calibration curve to the $45^{\circ}$ line in Figure S2 demonstrates excellent validation on an absolute probability scale. However, the extent of missing data casts some doubt on the validity of this model, and on the efficiency of its parameter estimates. ${ }^{21}$

\section{Predictors of CSS in patients with PTMC}

The mean length of follow-up was $43.5 \pm 29.9$ months and ranged from 0 to 107 months. During the follow-up period, only $63(0.2 \%)$ died as a result of a cancer-specific cause. Male sex, elder age ( $\geq 45$ years), gross ETE, LLNM, and distant metastasis were validated to be significant risk factors for cancer-specific death in both the univariate and multivariate Cox regression analyses. The patients with minimal ETE exhibited a significantly worse CSS compared with the patients without ETE; however, minimal ETE was not a significant predictor of cancer-specific death in the multivariate Cox regression analysis. Other factors, such as race, tumor size, histology, and multifocality, were not associated with CSS in univariate or multivariate analyses. In particular, TT or postoperative radioisotopes therapy did not improve the CSS significantly in patients with PTMC (Table 4).

\section{Discussion}

The incidence of PTC has increased rapidly in the past years; small PTCs account for most new diagnoses, which may result from the increased detection of small tumors that are incidentally noted on diagnostic imaging studies. ${ }^{1,22}$ Recently, the increased use of imaging procedures, such as ultrasonography of the neck, has identified a large number of small thyroid nodules, with a similar increasing trend in the proportion of PTMC in all PTC patients.

There were 31,017 patients $(40.9 \%)$ histologically diagnosed with PTMC based on the SEER data from 2002 to 2012. The prevalence rates of CLNM and LLNM in present study were $6.9 \%$ and $5.4 \%$, respectively, values that were lower compared to previous studies that reported a $17.8 \%-64.1 \%$ rate for CLNM and a $3.7 \%-44.5 \%$ rate for LLNM. ${ }^{4,8,23-30}$ The variation in the prevalence of cervical LNM across different studies may result from the differences in the number of patients, the different rationales (therapeutic or prophylactic) underlying the use of LND in PTMC, and the subtle variations in the surgical completeness among surgeons and the number of lymph nodes in histopathological findings detected among pathologists. ${ }^{15}$ 
Table 4 Univariate and multivariate Cox regression analysis of CSS in patients with PTMC

\begin{tabular}{|c|c|c|c|c|}
\hline \multirow[t]{2}{*}{ Independent variable } & \multicolumn{2}{|l|}{ Univariate } & \multicolumn{2}{|l|}{ Multivariate } \\
\hline & HR (95\% Cl) & $P$-value & HR (95\% Cl) & $P$-value \\
\hline Sex (female vs male) & $0.28 I(0.169-0.465)$ & 0.001 & $0.544(0.32 \mathrm{I}-0.923)$ & 0.024 \\
\hline Age ( $\geq 45$ vs $<45$ years) & $5.709(2.287-14.253)$ & 0.001 & $7.419(2.898-18.990)$ & 0.001 \\
\hline \multicolumn{5}{|l|}{ Race } \\
\hline Black & I (reference) & & I (reference) & \\
\hline White & $3.749(0.519-27.101)$ & 0.190 & $2.052(0.28 I-15.007)$ & 0.479 \\
\hline Other ${ }^{a}$ & $4.117(0.496-34.199)$ & 0.190 & $2.319(0.275-19.548)$ & 0.439 \\
\hline Size & $1.054(0.969-1.147)$ & 0.222 & $1.001(0.914-1.096)$ & 0.989 \\
\hline Histology (FV-PTMC vs PTMC) & $0.72 \mid(0.39|-| .33 \mid)$ & 0.295 & $0.845(0.452-1.577)$ & 0.596 \\
\hline Multifocality & $1.608(0.972-2.661)$ & 0.065 & $0.938(0.543-1.621)$ & 0.819 \\
\hline \multicolumn{5}{|l|}{ ETE } \\
\hline Negative & I (reference) & & I (reference) & \\
\hline Capsule & $0.759(0.104-5.513)$ & 0.785 & $0.625(0.085-4.564)$ & 0.643 \\
\hline Minimal & $3.262(1.531-6.948)$ & 0.002 & $1.358(0.604-3.053)$ & 0.458 \\
\hline Gross & $38.880(19.504-77.508)$ & 0.001 & $5.844(2.6|7-| 3.05 \mid)$ & 0.001 \\
\hline CLNM & $1.840(0.837-4.043)$ & 0.129 & $2.345(0.989-5.563)$ & 0.053 \\
\hline LLNM & $10.004(5.960-16.792)$ & 0.001 & $4.88 \mid(2.496-9.544)$ & 0.001 \\
\hline Metastasis $^{\mathrm{b}}$ & 85.31 I (45.373-160.404) & 0.001 & $|8.05|(8.732-37.3 \mid 4)$ & 0.001 \\
\hline TT & 1.318 (0.70I-2.479) & 0.391 & $0.794(0.396-1.595)$ & 0.518 \\
\hline Radioisotopes & $2.637(0.535-4.529)$ & 0.068 & $1.563(0.84 \mathrm{I}-2.904)$ & 0.158 \\
\hline
\end{tabular}

Notes: ancluding American Indian/Alaska Native and Asian/Pacific Islander. 'Including the lungs, bones, and brains.

Abbreviations: CSS, cancer-specific survival; PTMC, papillary thyroid microcarcinoma; HR, hazard ratio; Cl, confidence interval; FV-PTMC, follicular variant of papillary thyroid microcarcinoma; ETE, extrathyroidal extension; CLNM, central lymph node metastasis; LLNM, lateral lymph node metastasis; TT, total thyroidectomy.

In our study, multivariate logistic analyses were used to evaluate the risk factors for LNM. We determined that male sex, younger age $(<45$ years), non-African American race, increased tumor size, classical papillary histology, multifocality, and ETE were significantly associated with LNM; distant metastasis was also significantly associated with LLNM.

Male sex was a risk factor for LNM, which suggests that males require specialized neck checkups of the thyroid to enable the early detection of thyroid tumors. ${ }^{15}$ Patient age is known to be an important risk factor for patients with PTC $>1 \mathrm{~cm} ;{ }^{31,32}$ our results indicated that younger age ( $<45$ years) was associated with an increased risk of LNM in PTMC patients. In particular, children and adolescents $(<20$ years of age) are more likely to have more advanced nodal stage at the time of diagnosis. One large study found that $64 \%$ of children had cervical LNMs and $23 \%$ had distant metastases at the time of diagnosis. In contrast, studies of adults have found that up to $40 \%$ have LNMs and only $5 \%$ present with distant metastases. ${ }^{33}$ Despite the extent of disease at the time of diagnosis, younger patients generally have excellent survival rates. One large study found a $2 \%$ cause-specific mortality rate after 40 years of follow-up. ${ }^{33,34}$ This finding may be explained by the results of our survival analysis or current staging and risk-stratification systems. First, younger age was not associated with CSS according to our data; moreover, even when younger patients showed nodal involvement, they were still staged as I, and their risk of mortality did not increase. On the other hand, most authorities suggest that younger patients have been treated with TT and radioiodine, and rigorous initial surgical and radioiodine treatment followed by thyrotropin suppression has resulted in favorable outcomes. Therefore, younger age $(<45$ years) had a greater impact on LNM than on prognosis in PTMC patients.

A greater tumor size was significantly associated with LNM, which was consistent with a previous study. ${ }^{31}$ As noted, the evaluation of LLNM was not simply dependent on the primary tumor size but, more importantly, on the results of the preoperative imaging examination, especially in the PTMC patients with increased tumor size.

Interestingly, our data showed that the presence of LNM in papillary cancer follicular variant at diagnosis was unlikely to be PTC. This sub-variant histology has been consistently shown in many studies to be associated with increased mortality and poorer outcome; its wide invasiveness is probably owing to the distinct biological features of Hurthle cell carcinomas from PTC, such as the demonstration of vascular or capsular invasion and the metastatic spread to the blood.

Multifocal lesion and ETEs were also significant factors for LNM. A recent study suggested that multifocal lesion represented multiple lesions with individual characteristics; ${ }^{35}$ 
thus, the characteristics of the individual tumor, rather than multifocal lesion itself, tend to correlate more with the risk of LNM. Although ETE was not a common finding in PTMC, it could be well predicted with a preoperative, high-resolution ultrasound examination. ${ }^{36-38}$ A preoperative imaging examination helps to predict cervical LNM in PTMC patients. On the other hand, it is also important to differentiate the clinical significance of minimal ETE (American Thyroid Association [ATA] intermediate risk) from gross ETE of surrounding structures (ATA high risk) by pathology. ${ }^{28}$ The risk of recurrence associated with minimal ETE (pT3 disease manifest by ETE) ranges from 3\% to $9 \%$, while the risk of recurrence in patients with gross ETE (pT4a disease involving the subcutaneous soft tissues, larynx, trachea, esophagus, or recurrent laryngeal nerve) ranges from $23 \%$ to $40 \%{ }^{39-46}$

Based on our finding of the association between risk factors and LNM, we further designed a model that systematically considers multiple variables to estimate an individual risk of LNM in patients with PTMC. Our model employs easily accessible clinical information, with high concordance on an absolute probability scale. Coefficients from the model indicate a change in the $\log (\mathrm{OR})$ per unit increment in tumor size ( $1 \mathrm{~mm}$, as continuous variable) or presenting with risk factors (as binary variables), when they represent a single factor that is linear and does not interact with other factors and if all other factors are held constant. For either CLNM or LLNM, ETE had maximum weight in the predictive model. As a form of tumor invasion, ETE was consistent with the previously described risk factors in the criteria for the assessment of progression. On the other hand, the ATA developed consensus-based criteria for the assessment of recurrence risk, and this system has been validated retrospectively with clinical data. ${ }^{47}$ PTMC was divided into low-, intermediate-, and high-risk groups, which represented different risks of poor prognosis. The basis of the classification included risk factors such as local metastases (LNM), residual tumor, tumor invasion, and aggressive histology. Therefore, ETE and other risk factors listed by the ATA criteria might provide guidance in the initial management of PTMC patients.

The optimal management of PTMC is a controversial issue. Given the high incidence of multifocal lesion, extensive TT surgery was considered to be more likely to remove all disease foci and improve the prognosis. However, TT did not improve the prognosis in the present study. Namely, the evidence from the present study may favor lobectomy for most PTMC patients without risk factors because of the comparative impact of the prognosis and the lower rate of surgical morbidity reported compared with TT. ${ }^{48}$ As noted, the presence of distant metastases was a strong risk factor for cancer-specific death. TT and postoperative radioisotopes therapy were an important approach to detect positive distant metastases at diagnosis or recurrence during follow-up by imaging or whole-body scan. However, given the small number of patients who were presenting with radioactive iodine at diagnosis $(0.3 \%)$, it might be limited to perform postoperative radioisotopes therapy in all PTMC patients. Further studies are needed to explore the indication for RAI therapy in PTMC patients.

In the current guidelines, ${ }^{47,49,50}$ prophylactic CLND is not recommended for patients with clinically node-negative (cN0) PTMC. However, some authors have argued its application in $\mathrm{cN} 0 \mathrm{PTMC}$ because of the high prevalence of CLNM. ${ }^{25,27,35,51}$ The removal of the occult nodes in the central compartment may confirm the status of the central lymph node for predicting LLNM and guiding treatment, with the aim of reducing the disease persistence. ${ }^{52-55}$ Although CLNM was not significantly associated with prognosis according to survival analysis, from the viewpoint of the radical treatment of tumors, prophylactic CLND must be considered in PTMC patients who present with high-risk factors.

The rationale for prophylactic LLND in PTC is controversial. The relation between prophylactic LLND and locoregional control or survival has not been demonstrated in previous studies. In the present study, cervical LNM was a prognostic indicator in the PTMC patients, and similar results have also been demonstrated in several previous studies. ${ }^{56}$ However, the associations between the node status and the recurrence prognosis with a long-term follow-up period and in a larger PTMC population remain unknown. The majority of reports on the predictive factors associated with a high risk of node metastasis have included clinically lymph node-positive patients and have not addressed the issue of occult lateral lymph nodes. Lim et $\mathrm{al}^{57}$ conducted the first prospective quantitative evaluation of LLNM in PTMC without clinical LLNM. In their study, the incidence of occult LNM was as high as 55\%, and the primary tumor size and the CLNM were two important risk factors for LLNM. Therefore, it appears that prophylactic LLND may be recommended in selected patients with high-risk factors.

Our study has some limitations. First, the confirmation for LNM was mainly developed from the collection of therapeutic LNDs. The low incidence of LNM in this cohort suggests the possibility of underestimation of LNM in patients with PTMC. However, previous studies have 
shown that ultrasound is an important tool in the detection of metastatic nodes, with a high specificity and a positive predictive value. Therefore, the current ultrasound system is relatively diagnostically accurate for clinical LNM, and seems to be improved by our findings or the model from this study, which integrates a series of clinicopathologic variables. Second, in patients with LLNM from the SEER database, we could not differentiate the status of the central lymph node. Although CLNM is reported to predict LLNM, our risk evaluation for LLNM did not consider CLNM as a risk factor in the multivariate analysis. Diagnostic algorithms for LNM suggested by this analysis have not been tested and will require prospective study before recommendations can be made. We also recommend prospective multicenter studies with long-term follow-ups to ascertain the optimal surgical approach for patients with PTMC.

In conclusion, the results of our study demonstrate that male sex, younger age $(<45$ years $)$, white and other race (including American Indian/AK Native, Asian/Pacific Islander), a greater tumor size, ETE, and multifocality are risk factors for cervical LNM. Distant metastasis is also a predictor of LLNM. CLND is recommended in select patients with risk factors. Surgeons could identify some of the high-risk factors or features which are associated with LLNM preoperatively by ultrasound, imaging exams, fineneedle biopsy, or molecular analysis. Given the prevalence of LLNM in PTMC, a thorough inspection of the lateral compartment is recommended in PTMC patients with risk factors; from the viewpoint of radical treatment for tumors, prophylactic LLND that aims to remove occult lateral lymph nodes may be an option for PTMC with high-risk factors. We recommend prospective multicenter studies with longterm follow-ups to ascertain the optimal surgical approach for patients with PTMC.

\section{Acknowledgments}

The authors acknowledge the efforts of the SEER Program tumor registries in the creation of the SEER database. The interpretation and reporting of these data are the sole responsibility of the authors. This work was supported by funds from the National Science Foundation of China (No 81572622 and No 81272934 to QHJ).

\section{Disclosure}

The authors declare that there is no conflict of interest that could be perceived as prejudicing the impartiality of the research reported.

\section{References}

1. McLeod DS, Sawka AM, Cooper DS. Controversies in primary treatment of low-risk papillary thyroid cancer. Lancet. 2013;381(9871): 1046-1057.

2. Wartofsky L. Management of papillary microcarcinoma: primum non nocere? J Clin Endocrinol Metab. 2012;97(4):1169-1172.

3. Shindo M, Wu JC, Park EE, Tanzella F. The importance of central compartment elective lymph node excision in the staging and treatment of papillary thyroid cancer. Arch Otolaryngol Head Neck Surg. 2006;132(6):650-654.

4. Wada N, Duh QY, Sugino K, et al. Lymph node metastasis from 259 papillary thyroid microcarcinomas: frequency, pattern of occurrence and recurrence, and optimal strategy for neck dissection. Ann Surg. 2003; 237(3):399-407.

5. Chow SM, Law SC, Chan JK, Au SK, Yau S, Lau WH. Papillary microcarcinoma of the thyroid-prognostic significance of lymph node metastasis and multifocality. Cancer. 2003;98(1):31-40.

6. Ito Y, Tomoda $\mathrm{C}$, Uruno $\mathrm{T}$, et al. Papillary microcarcinoma of the thyroid: how should it be treated? World J Surg. 2004;28(11):1115-1121.

7. Hay ID, Hutchinson ME, Gonzalez-Losada T, et al. Papillary thyroid microcarcinoma: a study of 900 cases observed in a 60-year period. Surgery. 2008;144(6):980-987; discussion 987-988.

8. So YK, Son YI, Hong SD, et al. Subclinical lymph node metastasis in papillary thyroid microcarcinoma: a study of 551 resections. Surgery. 2010;148(3):526-531.

9. Ito $\mathrm{Y}$, Tomoda $\mathrm{C}$, Uruno $\mathrm{T}$, et al. Clinical significance of metastasis to the central compartment from papillary microcarcinoma of the thyroid. World J Surg. 2006;30(1):91-99.

10. Pelizzo MR, Boschin IM, Toniato A, et al. Natural history, diagnosis, treatment and outcome of papillary thyroid microcarcinoma (PTMC): a mono-institutional 12-year experience. Nucl Med Commun. 2004;25(6):547-552.

11. Bramley MD, Harrison BJ. Papillary microcarcinoma of the thyroid gland. Br J Surg. 1996;83(12):1674-1683.

12. Malandrino P, Pellegriti G, Attard M, et al. Papillary thyroid microcarcinomas: a comparative study of the characteristics and risk factors at presentation in two cancer registries. J Clin Endocrinol Metab. 2013; 98(4):1427-1434.

13. Roh JL, Kim JM, Park CI. Central cervical nodal metastasis from papillary thyroid microcarcinoma: pattern and factors predictive of nodal metastasis. Ann Surg Oncol. 2008;15(9):2482-2486.

14. Ducoudray R, Tresallet C, Godiris-Petit G, Tissier F, Leenhardt L, Menegaux F. Prophylactic lymph node dissection in papillary thyroid carcinoma: is there a place for lateral neck dissection? World J Surg. 2013; 37(7):1584-1591.

15. Qu N, Zhang L, Ji QH, et al. Risk factors for central compartment lymph node metastasis in papillary thyroid microcarcinoma: a meta-analysis. World J Surg. 2015;39(10):2459-2470.

16. Warren JL, Klabunde CN, Schrag D, Bach PB, Riley GF. Overview of the SEER-Medicare data: content, research applications, and generalizability to the United States elderly population. Med Care. 2002; 40(8 Suppl):IV-3-18.

17. Edge SB, Compton CC. The American Joint Committee on Cancer: the 7th edition of the AJCC cancer staging manual and the future of TNM. Ann Surg Oncol. 2010;17(6):1471-1474.

18. Sobin LH, Gospodarowicz MK, Wittekind CH. UICC: TNM Classification of Malignant Tumors. 7th ed. New York: Wiley-Liss; 2009.

19. Harrell FE Jr, Lee KL, Mark DB. Multivariable prognostic models: issues in developing models, evaluating assumptions and adequacy, and measuring and reducing errors. Stat Med. 1996;15(4):361-387.

20. Nunez E, Steyerberg EW, Nunez J. Regression modeling strategies. Rev Esp Cardiol. 2011;64(6):501-507.

21. Frank E, Harrell J. Regression modeling strategies. In: Logistic Model Case Study 2: Survival of Titanic Passengers. 2nd ed. New York: Springer; 2001:291-310. 
22. Siegel R, Naishadham D, Jemal A. Cancer statistics, 2013. CA Cancer J Clin. 2013;63(1):11-30.

23. Lee J, Park JH, Lee CR, Chung WY, Park CS. Long-term outcomes of total thyroidectomy versus thyroid lobectomy for papillary thyroid microcarcinoma: comparative analysis after propensity score matching. Thyroid. 2013;23(11):1408-1415.

24. Zhou YL, Gao EL, Zhang W, et al. Factors predictive of papillary thyroid micro-carcinoma with bilateral involvement and central lymph node metastasis: a retrospective study. World J Surg Oncol. 2012;10:67.

25. Moon HJ, Kim EK, Yoon JH, Kwak JY. Clinical implication of elastography as a prognostic factor of papillary thyroid microcarcinoma. Ann Surg Oncol. 2012;19(7):2279-2287.

26. Hyun SM, Song HY, Kim SY, et al. Impact of combined prophylactic unilateral central neck dissection and hemithyroidectomy in patients with papillary thyroid microcarcinoma. Ann Surg Oncol. 2012;19(2): 591-596.

27. Caliskan M, Park JH, Jeong JS, et al. Role of prophylactic ipsilateral central compartment lymph node dissection in papillary thyroid microcarcinoma. Endocr J. 2012;59(4):305-311.

28. Moon HJ, Kim EK, Chung WY, Yoon JH, Kwak JY. Minimal extrathyroidal extension in patients with papillary thyroid microcarcinoma: is it a real prognostic factor? Ann Surg Oncol. 2011;18(7):1916-1923.

29. Lee KJ, Cho YJ, Kim SJ, et al. Analysis of the clinicopathologic features of papillary thyroid microcarcinoma based on 7-mm tumor size. World J Surg. 2011;35(2):318-323.

30. Kim TY, Hong SJ, Kim JM, et al. Prognostic parameters for recurrence of papillary thyroid microcarcinoma. BMC Cancer. 2008;8:296.

31. Zhang L, Wei WJ, Ji QH, et al. Risk factors for neck nodal metastasis in papillary thyroid microcarcinoma: a study of 1066 patients. J Clin Endocrinol Metab. 2012;97(4):1250-1257.

32. Koo BS, Choi EC, Yoon YH, Kim DH, Kim EH, Lim YC. Predictive factors for ipsilateral or contralateral central lymph node metastasis in unilateral papillary thyroid carcinoma. Ann Surg. 2009;249(5):840-844.

33. Adam MA, Pura J, Goffredo P, et al. Presence and number of lymph node metastases are associated with compromised survival for patients younger than age 45 years with papillary thyroid cancer. J Clin Oncol. 2015; 33(21):2370-2375.

34. Ganly I, Nixon IJ, Wang LY, et al. Survival from differentiated thyroid cancer: what has age got to do with it? Thyroid. 2015;25(10): 1106-1114.

35. Lee SH, Lee SS, Jin SM, Kim JH, Rho YS. Predictive factors for central compartment lymph node metastasis in thyroid papillary microcarcinoma. Laryngoscope. 2008;118(4):659-662.

36. Moon SJ, Kim DW, Kim SJ, Ha TK, Park HK, Jung SJ. Ultrasound assessment of degrees of extrathyroidal extension in papillary thyroid microcarcinoma. Endocr Pract. 2014;20(10):1037-1043.

37. Kwak JY, Kim EK, Youk JH, et al. Extrathyroid extension of welldifferentiated papillary thyroid microcarcinoma on US. Thyroid. 2008; 18(6):609-614.

38. Gweon HM, Son EJ, Youk JH, Kim JA, Park CS. Preoperative assessment of extrathyroidal extension of papillary thyroid carcinoma: comparison of 2- and 3-dimensional sonography. J Ultrasound Med. 2014; 33(5):819-825.

39. Parsons JT, Mendenhall WM. Treatment of extrathyroidal extension from papillary thyroid cancer. Head Neck. 1995;17(1):73.

40. Hu A, Clark J, Payne RJ, Eski S, Walfish PG, Freeman JL. Extrathyroidal extension in well-differentiated thyroid cancer: macroscopic vs microscopic as a predictor of outcome. Arch Otolaryngol Head Neck Surg. 2007;133(7):644-649.
41. Radowsky JS, Howard RS, Burch HB, Stojadinovic A. Impact of degree of extrathyroidal extension of disease on papillary thyroid cancer outcome. Thyroid. 2014;24(2):241-244.

42. Lee CY, Kim SJ, Ko KR, Chung KW, Lee JH. Predictive factors for extrathyroidal extension of papillary thyroid carcinoma based on preoperative sonography. J Ultrasound Med. 2014;33(2):231-238.

43. Ahn D, Sohn JH, Jeon JH, Jeong JY. Clinical impact of microscopic extrathyroidal extension in patients with papillary thyroid microcarcinoma treated with hemithyroidectomy. J Endocrinol Invest. 2014; 37(2):167-173.

44. Jung SP, Kim M, Choe JH, Kim JS, Nam SJ, Kim JH. Clinical implication of cancer adhesion in papillary thyroid carcinoma: clinicopathologic characteristics and prognosis analyzed with degree of extrathyroidal extension. World J Surg. 2013;37(7):1606-1613.

45. Sia MA, Tsang RW, Panzarella T, Brierley JD. Differentiated thyroid cancer with extrathyroidal extension: prognosis and the role of external beam radiotherapy. $J$ Thyroid Res. 2010;2010:183461.

46. Lombardi CP, Bellantone R, De Crea C, et al. Papillary thyroid microcarcinoma: extrathyroidal extension, lymph node metastases, and risk factors for recurrence in a high prevalence of goiter area. World J Surg. 2010;34(6):1214-1221.

47. Haugen BRM, Alexander EK, Bible KC, et al. 2015 American Thyroid Association management guidelines for adult patients with thyroid nodules and differentiated thyroid cancer. Thyroid. 2016;26(1):1-133.

48. Lim YC, Choi EC, Yoon YH, Kim EH, Koo BS. Central lymph node metastases in unilateral papillary thyroid microcarcinoma. Br J Surg. 2009;96(3):253-257.

49. Cooper DS, Doherty GM, Haugen BR, et al. Revised American Thyroid Association management guidelines for patients with thyroid nodules and differentiated thyroid cancer. Thyroid. 2009;19(11):1167-1214.

50. Pitoia F, Miyauchi A. 2015 American Thyroid Association guidelines for thyroid nodules and differentiated thyroid cancer and their implementation in various care settings. Thyroid. 2016;26(2):319-321.

51. Kim BY, Jung CH, Kim JW, et al. Impact of clinicopathologic factors on subclinical central lymph node metastasis in papillary thyroid microcarcinoma. Yonsei Med J. 2012;53(5):924-930.

52. Suman P, WangCH, Abadin SS, Moo-Young TA, PrinzRA, WinchesterDJ. Risk factors for central lymph node metastasis in papillary thyroid carcinoma: a National Cancer Data Base (NCDB) study. Surgery. 2016;159(1):31-39.

53. Xu JM, Xu XH, Xu HX, et al. Prediction of cervical lymph node metastasis in patients with papillary thyroid cancer using combined conventional ultrasound, strain elastography, and acoustic radiation force impulse (ARFI) elastography. Eur Radiol. 2016;26(8):2611-2622.

54. Lee YS, Lim YS, Lee JC, et al. Ultrasonographic findings relating to lymph node metastasis in single micropapillary thyroid cancer. World J Surg Oncol. 2014;12:273.

55. Agarwal S, Chand G, Jaiswal S, et al. Pattern and risk factors of central compartment lymph node metastasis in papillary thyroid cancer: a prospective study from an endocrine surgery centre. J Thyroid Res. 2012;2012:436243.

56. Ito Y, Fukushima M, Tomoda C, et al. Prognosis of patients with papillary thyroid carcinoma having clinically apparent metastasis to the lateral compartment. Endocr J. 2009;56(6):759-766.

57. Lim YS, Lee JC, Lee YS, et al. Lateral cervical lymph node metastases from papillary thyroid carcinoma: predictive factors of nodal metastasis. Surgery. 2011;150(1):116-121. 


\section{Supplementary materials}
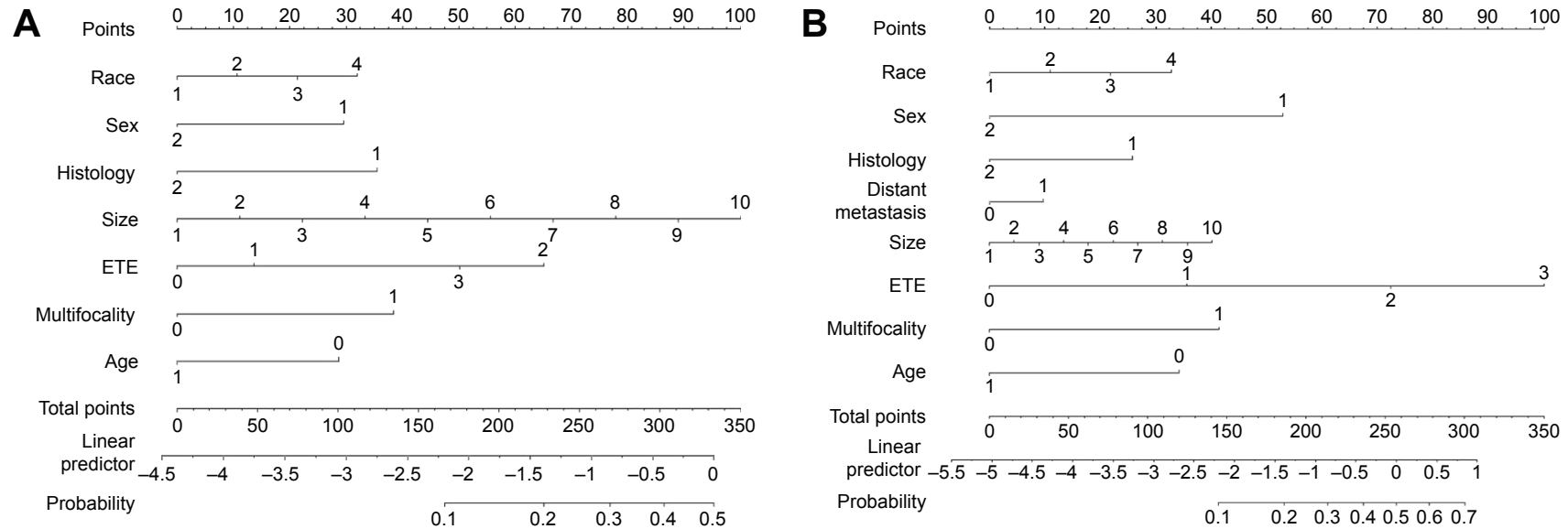

Figure SI Lymph node metastasis predictive model.

Notes: A model selection was performed by a backward stepdown selection process with the Akaike information criterion. (A) Central lymph node metastasis. (B) Lateral lymph node metastasis.

Abbreviation: ETE, extrathyroidal extension.
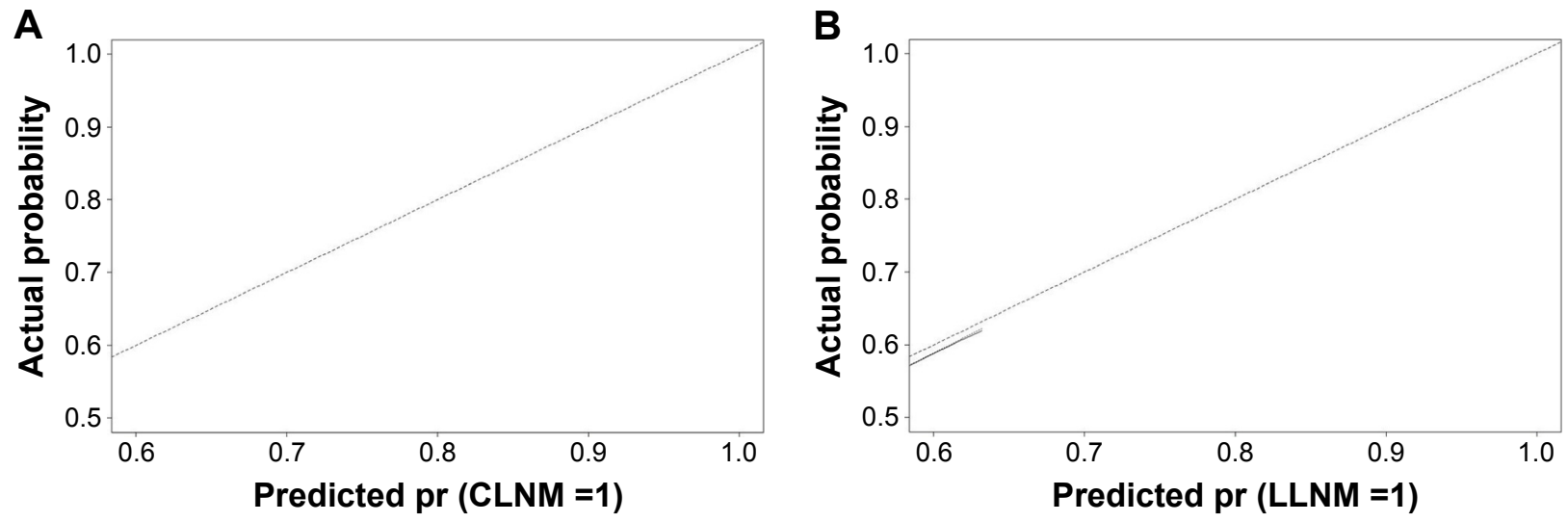

Apparent - Bias-corrected -.. Ideal

Figure S2 The validation for the model by the calibration curve.

Notes: (A) Central lymph node metastasis. (B) Lateral lymph node metastasis.

Abbreviations: CLNM, central lymph node metastasis; LLNM, lateral lymph node metastasis.

\section{Publish your work in this journal}

OncoTargets and Therapy is an international, peer-reviewed, open access journal focusing on the pathological basis of all cancers, potential targets for therapy and treatment protocols employed to improve the management of cancer patients. The journal also focuses on the impact of management programs and new therapeutic agents and protocols on patient perspectives such as quality of life, adherence and satisfaction. The manuscript management system is completely online and includes a very quick and fair peer-review system, which is all easy to use. Visit http://www.dovepress.com/testimonials.php to read real quotes from published authors. 